\title{
Stability of the pulselike solutions of the quintic complex Ginzburg-Landau equation
}

\author{
J. M. Soto-Crespo \\ Instituto de Óptica, Consejo Superior de Investigaciones Científicas, Serrano 121, 28006 Madrid, Spain
}

\author{
N. N. Akhmediev and V. V. Afanasjev \\ Optical Sciences Centre, Institute of Advanced Studies, \\ The Australian National University, Canberra, 0200, Australia
}

Received July 31, 1995; revised manuscript received November 7, 1995

\begin{abstract}
We performed a detailed investigation of the stability of analytic pulselike solutions of the quintic complex Ginzburg-Landau equation that describes the dynamics of the field in a passively mode-locked laser. We found that in general they are unstable except in a few special cases. We also obtained regions in the parameter space in which stable pulse solutions exist. These stable solutions do not have analytical expressions and must be calculated numerically. We compared and connected the regions in which stable solitonlike solutions exist with the lines for which we had analytical solutions. (c) 1996 Optical Society of America
\end{abstract}

\section{INTRODUCTION}

The cubic-quintic complex Ginzburg-Landau equation (CGLE) is a continuous approximation to the dynamics of the field in a passively mode-locked laser. ${ }^{1-3}$ It has also been proven to be useful in describing important phenomena such as ultrashort pulse propagation in optical transmission lines with spectral filtering ${ }^{4-9}$ and erbium-doped fiber amplifiers. ${ }^{10}$ Earlier, the CGLE was used successfully in modeling of other nonequilibrium processes in physics. ${ }^{11-13}$ The quintic terms in the equation describe important physics, which is lacking in other models in the literature. ${ }^{1,14}$ The most important, the quintic CGLE, has stable pulselike solutions. We recall that pulselike solutions of the cubic CGLE are unstable in general. ${ }^{15}$

Of crucial importance is to know for which values of the coefficients the quintic CGLE has stable pulselike solutions. ${ }^{16}$ The best way to answer this question is to find its exact solutions and to study their stability. However, exact solutions have been found analytically only when special relations between the parameters of the equations are satisfied. Otherwise, the solutions must be studied numerically or with certain approximations. ${ }^{17,18}$

Exact solutions of the quintic CGLE have been studied by van Saarloos and Hohenberg ${ }^{19,20}$ and in a recent study by Marcq, Chaté, and Conte. ${ }^{2}$ The method used in Refs. 19 and 20 is the reduction of the original partial differential equations into a set of three ordinary differential equations that admits exact solutions. However, solutions have not been written explicitly. The authors of Ref. 21 used Painlevé analysis and symbolic computations. Thus far this study constitutes the most comprehensive mathematical treatment of the quintic CGLE. The general approach, used in Ref. 21, consists of the reduction of the differential equation to a purely algebraic problem. However, this technique assumes that analytical results can be obtained in a reasonable time only by use of computers. More importantly, the final formula for the pulselike solutions in Ref. 21 has parameters that are expressed implicitly through the coefficients of the CGLE, and thus some work is still needed if we are to calculate the pulse shapes numerically.

In this paper we rederive exact pulselike solutions of the quintic CGLE in a simpler way, obtaining these solutions in analytical form, and, in addition, we study their stability. Note that the stability of the pulselike solutions of the quintic CGLE is still an open question. Only for the case of the cubic CGLE has it been investigated. ${ }^{3}$ We found that exact analytic solutions of the quintic CGLE are unstable everywhere in the region of parameters in which they exist. An exception occurs when the pulse is in the vicinity of the zone in which it is transformed into two zero-velocity fronts. The solution then looks like a flat-top soliton.

As a next step, we investigate numerically the whole range of parameters in which we would expect that stable solitonlike pulses exist. As a result we found the area of global stable pulse propagation, i.e., the region in the parameter space in which a broad class of initial conditions converge to a stationary pulse, which therefore represents a stable pulselike solution of the quintic CGLE. This should be very useful for experimental researchers working in this field. In principle, the CGLE has different types of solutions, including pulses, fronts, sinks, and sources. ${ }^{12,19-22}$ In this paper we restrict ourselves to pulselike solutions, as they are the most important ones for optical applications.

In the field of nonlinear optics the quintic CGLE is usually written in the following form:

$$
\begin{aligned}
i \psi_{z}+\frac{1}{2} \psi_{t t}+|\psi|^{2} \psi= & i \delta \psi+i \epsilon|\psi|^{2} \psi+i \beta \psi_{t t} \\
& +i \mu|\psi|^{4} \psi-\nu|\psi|^{4} \psi,
\end{aligned}
$$

where $t$ is the retarded time, $z$ is the propagation distance, $\delta$ is the linear excess gain at the carrier frequency, 
$\beta$ describes spectral filtering $(\beta>0), \epsilon$ accounts for nonlinear gain/absorption processes, $\mu$ represents a higherorder correction to the nonlinear amplification/absorption, and $\nu$ is a higher-order correction term to the nonlinear refractive index. All coefficients in Eq. (1) are real constants (we do not require them to be small), and $\psi$ is the complex envelope of the electric field. Equation (1) is written in such a way that, if the right-hand side of it is set to zero, we obtain the standard nonlinear Schrödinger equation.

Note that $\beta$ must be nonnegative in order to stabilize the pulse in the frequency domain. If the coefficients $\delta$, $\beta, \epsilon$, and $\nu$ on the right-hand side are small and $\nu=0$, then solitonlike solutions of Eq. (1) can be studied by application of perturbative theory to the soliton solutions of the nonlinear Schrödinger equation. ${ }^{1,4,5}$ This approach, however, can give neither all the relevant properties of solitonlike pulses nor the regions in the parameter space in which they exist. Finding exact solutions is an important step for understanding the full range of properties of the complex CGLE, thus helping us to predict the behavior resulting from an arbitrary initial condition.

The structure of the rest of the paper is the following. In Section 2 we rederive exact solutions of the quintic CGLE, whose stability is analyzed in Section 3, in which we also show some examples of propagation of different inputs. In Section 4 we study numerically the regions in the parameter space in which stable stationary pulses are possible. The form of these pulses cannot be expressed analytically yet, but there is a clear relation between the subset of parameters for which we obtained analytical solutions and that in which stable pulses are possible. This indicates that, although the analytical solutions are defined in a subspace of the full parameter space, they play an important role in understanding the dynamics of a general solution. This is dealt with in Section 5 . Finally, Section 6 summarizes our results.

\section{ANALYTICAL PROCEDURES}

In this study we are interested in pulselike solutions of Eq. (1) that have zero transverse velocity. Hence we look for a solution of the form

$$
\psi(t, z)=A(t) \exp (-i \omega z)
$$

where $\omega$ is a real constant. The complex function $A(t)$ can always be written in an explicit form as

$$
A(t)=a(t) \exp [i \phi(t)]
$$

where $a$ and $\phi$ are real functions of $t$. By inserting Eqs. (2) and (3) into Eq. (1) and separating real and imaginary terms, we obtain

$$
\begin{aligned}
& \left(\omega-\frac{1}{2} \phi^{2}+\beta \phi^{\prime \prime}\right) a+2 \beta \phi^{\prime} a^{\prime}+\frac{1}{2} a^{\prime \prime}+a^{3}+\nu a^{5}=0, \\
& \left(-\delta+\beta \phi^{\prime 2}+\frac{1}{2} \phi^{\prime \prime}\right) a+\phi^{\prime} a^{\prime}-\beta a^{\prime \prime}-\epsilon a^{3}-\mu a^{5}=0,
\end{aligned}
$$

where a prime stands for differentiation with respect to $t$.
Let us now assume that

$$
\phi(t)=\phi_{0}+d \ln [a(t)]
$$

where $d$ is the chirp parameter and $\phi_{0}$ is an arbitrary phase; we suppose $\phi_{0}=0$ for simplicity. Equation (5) is, obviously, a restriction imposed on $\phi(t)$ because the chirp could have a more general functional dependence on $t$. However, this restriction allows us to find some families of solutions in analytical form. The solutions studied in this section are only those that can be represented in the form of Eqs. (3) and (5). Equations (4) then become

$$
\begin{aligned}
& \omega a+\left(\frac{1}{2}+\beta d\right) a^{\prime \prime}+\left(\beta d-\frac{d^{2}}{2}\right) \frac{a^{\prime 2}}{a}+a^{3}+\nu a^{5}=0 \\
& -\delta a+\left(\frac{d}{2}-\beta\right) a^{\prime \prime}+\left(\frac{d}{2}+\beta d^{2}\right) \frac{a^{\prime 2}}{a}-\epsilon a^{3}-\mu a^{5}=0 .
\end{aligned}
$$

Now, we have two second-order ordinary differential equations relative to the same dependent variable, $a(t)$. To have a common solution, the two equations must be compatible. In general, this is not the case. However, for this particular system, they can be made compatible by a proper choice of parameters. To find the conditions of compatibility, we use the following procedure.

We eliminate the first derivatives from the set of Eqs. (6) and integrate the resulting equation, obtaining

$$
\begin{aligned}
\frac{d}{4}\left(1+d^{2}\right)( & \left.1+4 \beta^{2}\right) \frac{a^{\prime 2}}{a^{2}}+\frac{1}{2}\left(\frac{d}{2}+\beta d^{2}+\epsilon \beta d-\frac{\epsilon d^{2}}{2}\right) a^{2} \\
+ & \frac{1}{3}\left[\nu\left(\frac{d}{2}+\beta d^{2}\right)+\mu\left(\beta d-\frac{d^{2}}{2}\right)\right] a^{4} \\
+ & \frac{\omega d}{2}(1+2 \beta d)+\delta\left(\beta d-\frac{d^{2}}{2}\right)=0 .
\end{aligned}
$$

The integration constant is zero for solutions decreasing to zero at infinity.

In contrast, we can eliminate the second derivative from Eqs. (6), obtaining

$$
\begin{aligned}
\frac{d}{4}\left(1+d^{2}\right)\left(1+4 \beta^{2}\right) \frac{a^{\prime 2}}{a^{2}}+\left(\beta-\frac{d}{2}-\frac{\epsilon}{2}-\epsilon \beta d\right) a^{2} \\
-\left[\nu\left(\frac{d}{2}-\beta\right)+\mu\left(\beta d+\frac{1}{2}\right)\right] a^{4} \\
+\omega \beta-\frac{\omega d}{2}-\frac{\delta}{2}-\delta \beta d=0 .
\end{aligned}
$$

These last two equations must coincide. Hence the following set of three algebraic equations must be satisfied:

$$
\begin{gathered}
\nu\left(4 d+2 \beta d^{2}-6 \beta\right)+\mu\left(8 \beta d-d^{2}+3\right)=0, \\
3 d+2 \beta d^{2}-4 \beta+6 \epsilon \beta d+2 \epsilon-\epsilon d^{2}=0, \\
2 \omega\left(d-\beta+\beta d^{2}\right)+\delta\left(1-d^{2}+4 \beta d\right)=0 .
\end{gathered}
$$

Equations (9) are the conditions of compatibility for Eqs. (6). 
If both coefficients $\mu$ and $\nu$ are nonzero, then Eq. (9a) gives the relation between the four parameters $\epsilon, \beta, \mu$, and $\nu$ when the solution exists in the form of Eqs. (3) and (5). The parameter $d$ can be found from Eq. (9b):

$$
d=d_{ \pm}=\frac{3(1+2 \epsilon \beta) \pm \sqrt{9(1+2 \epsilon \beta)^{2}+8(\epsilon-2 \beta)^{2}}}{2(\epsilon-2 \beta)}
$$

This is an important result, which shows that $d$ can be found in terms of $\beta$ and $\epsilon$ only.

From Eq. (9c) we obtain for $\omega$ :

$$
\omega=-\frac{\delta\left(1-d^{2}+4 \beta d\right)}{2\left(d-\beta+\beta d^{2}\right)} .
$$

Now, taking into account Eqs. (9a), (10), and (11), and after some cumbersome transformations, we can rewrite Eq. (8) [or Eq. (7)] in the form

$$
\begin{gathered}
\frac{a^{\prime 2}}{a^{2}}+\frac{2 \nu}{8 \beta d-d^{2}+3} a^{4}+\frac{2(2 \beta-\epsilon)}{3 d\left(1+4 \beta^{2}\right)} a^{2} \\
-\frac{\delta}{d-\beta+\beta d^{2}}=0
\end{gathered}
$$

The coefficient in front of $a^{4}$ can equally be written in the form

$$
\frac{2 \nu}{8 \beta d-d^{2}+3}=\frac{\mu}{3 \beta-2 d-\beta d^{2}} .
$$

By using the substitution $\mathrm{f}=\mathrm{a}^{2}$, we can rewrite Eq. (12) in the form

$$
\begin{gathered}
\frac{f^{\prime 2}}{f^{2}}+\frac{8 \nu}{8 \beta d-d^{2}+3} f^{2}+\frac{8(2 \beta-\epsilon)}{3 d\left(1+4 \beta^{2}\right)} f \\
-\frac{4 \delta}{d-\beta+\beta d^{2}}=0 .
\end{gathered}
$$

It is important to note that Eq. (14) is the consequence of set (6), and its solutions are equivalent to the solutions of set (6). Equation (14) is an elliptic equation, and its solutions can be found relatively easily. Bounded solitonlike solutions exist only if

$$
\frac{4 \delta}{d-\beta+\beta d^{2}}>0 \text {. }
$$

If we take $d=d_{-}$, which as is shown below is the most interesting value, the denominator in this expression is positive below the curve $S$, given by

$$
\epsilon_{S}=\beta \frac{3 \sqrt{1+4 \beta^{2}}-1}{4+18 \beta^{2}},
$$

and is negative above it (see the solid curve in Fig. 1). Hence if $\delta$ is negative, we can have a solution above curve (16), and below it if $\delta$ is positive.

The positive solution of Eq. (14) is ${ }^{23}$

$$
f(t)=\frac{2 f_{1} f_{2}}{\left(f_{1}+f_{2}\right)-\left(f_{1}-f_{2}\right) \cosh \left(2 \alpha \sqrt{f_{1}\left|f_{2}\right|} t\right)},
$$

where

$$
\alpha=\sqrt{\left|\frac{2 \nu}{8 \beta d-d^{2}+3}\right|}=\sqrt{\left|\frac{\mu}{3 \beta-2 d-\beta d^{2}}\right|},
$$

and $f_{1}$ and $f_{2}$ are the roots of the equation

$$
\frac{2 \nu}{8 \beta d-d^{2}+3} f^{2}+\frac{2(2 \beta-\epsilon)}{3 d\left(1+4 \beta^{2}\right)} f-\frac{\delta}{d-\beta+\beta d^{2}}=0
$$

namely,

$$
\begin{aligned}
& f_{1,2}= \\
& \frac{-(2 \beta-\epsilon) \pm \sqrt{(2 \beta-\epsilon)^{2}+\frac{18 \delta d^{2} \nu\left(1+4 \beta^{2}\right)^{2}}{\left(8 \beta d-d^{2}+3\right)\left(d-\beta+\beta d^{2}\right)}}}{6 d \nu\left(1+4 \beta^{2}\right)} \\
& \times\left(8 \beta d-d^{2}+3\right), \quad(20)
\end{aligned}
$$

When $\nu=0$, this expression must be replaced by

$$
\begin{aligned}
& f_{1,2}= \\
& \frac{-(2 \beta-\epsilon) \pm \sqrt{(2 \beta-\epsilon)^{2}+\frac{9 \delta d^{2} \mu\left(1+4 \beta^{2}\right)^{2}}{\left(3 \beta-2 d-\beta d^{2}\right)\left(d-\beta+\beta d^{2}\right)}}}{3 d \mu\left(1+4 \beta^{2}\right)} \\
& \times\left(3 \beta-2 d-\beta d^{2}\right) \quad(21)
\end{aligned}
$$

Apart from the necessary condition (15), the above soliton solution (17) exists if one of the roots $f_{1,2}$ is positive. Taking these two conditions in mind, we can obtain the region of existence of these solutions very easily numerically.

The soliton solutions of the quintic CGLE exist for a wide range of values of the coefficients $\beta, \epsilon, \mu$, and $\nu$. The ansatz (5) is the condition that restricts this range by imposing the relation [Eq. (9a)] on them. The general solution exists for both signs in expression (10) for $d$. This means that solutions must be analyzed separately in the two cases.

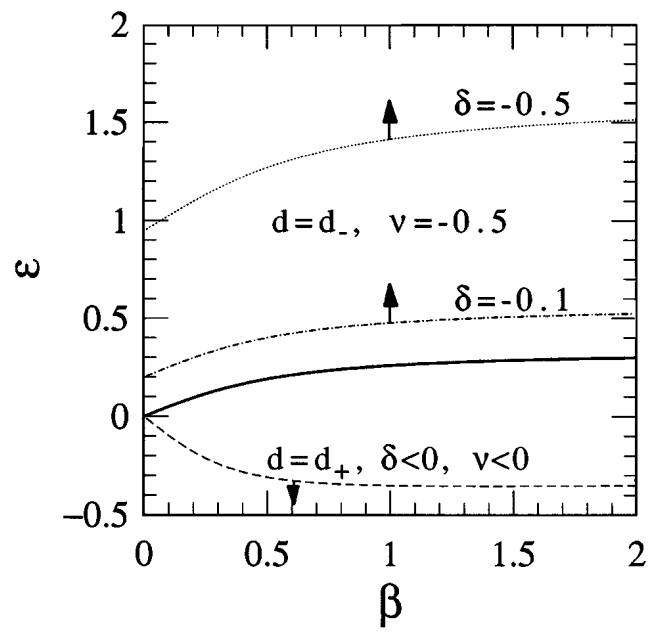

Fig. 1. Curves delimiting the regions on the $(\beta, \epsilon)$ plane for which the stationary solutions [Eq. (21)] studied in this paper exist. The dotted-dashed and the dotted curves are obtained when $d=d_{-}$and $\nu=-0.5$ and when $\delta=-0.1$ and $\delta=-0.5$, respectively. The region in which the solution exists is the area above the curves. The dashed curve is for $d=d_{+}$and for negative values of $\delta$ and $\nu$. The allowed region is in this last case the area below the curve. The solid curve represents the curve given by Eq. (16). 
As an illustrative example, Fig. 1 shows three curves that delimit the region of allowed values of the parameters $(\beta, \epsilon)$ in which the solution exists for given values of $\nu$ and $\delta$. The dotted-dashed and the dotted curves are obtained for $\nu=-0.5, d=d_{-}$, and for $\delta=-0.1$ and $\delta=-0.5$, respectively. The region above each curve represents the allowed values of the parameters $(\beta, \epsilon)$ in which the solution exists in each case. $\delta$ and $\nu$ being both negative, the corresponding curve is the same if the product $\delta \nu$ is kept constant. The dashed curve shows the same, but now for $d=d_{+}$. In this case the region of allowed values of $(\beta, \epsilon)$ is the area located below the curve, and as far as the signs of $\nu$ or $\delta$ do not change, it depends only on product $\delta \nu$.

Given an arbitrary selection of three parameters (let us choose $\beta, \nu$, and $\delta$ ), the stationary solution (21) exists for a certain range of values of $\epsilon$. The parameter $\mu$ is then determined by Eq. (9a) to have two possible values corresponding to the two possible values of $d=d_{ \pm}$. Figure 2 shows the dependence of $\mu$ with respect to $\epsilon$ as given by Eq. (9a) for $\beta=0.5$ and $\nu=-0.5$. The continuous curve is the one obtained by choice of $d=d_{+}$in Eq. (10), and the dotted curve is for $d=d_{-}$. The horizontal lines mark the allowed values of $\epsilon$ at which the solution exists for a fixed value of $\delta$, which is stated on the lines together with the corresponding choice for $d$. Similar couples of curves $\mu(\epsilon)$ for $d=d_{-}$and $d=d_{+}$can be plotted for other values of $\beta$ and $\nu$. Then the value of $\delta$ delimits the values of $\epsilon$ at which the analytical solution exists.

\section{LINEAR STABILITY ANALYSIS}

To investigate the stability of any of the solutions, we seek a perturbed solution of the form

$$
\psi(t, z)=\left[A_{0}(t)+\gamma g(t, z)\right] \exp (-i \omega z),
$$

where $A_{0}(t)$ is the stationary solution under study [see Eq. (3)], $\gamma$ is a small parameter, and $g(z, t)$ is a perturbation function. Inserting Eq. (22) into Eq. (1) and linearizing in the small parameter $\gamma$, we obtain

$$
\begin{gathered}
i g_{z}+\omega g+\left(\frac{1}{2}-i \beta\right) g_{t t}+2\left|A_{0}\right|^{2}(1-i \epsilon) g \\
+A_{0}^{2}(1-i \epsilon) g^{*}+(\nu-i \mu)\left(3\left|A_{0}\right|^{4} g+2\left|A_{0}\right|^{2} A_{0}^{2} g^{*}\right)=0 .
\end{gathered}
$$

This equation for the perturbation function $g$ has potentially many possible types of solutions. Moreover, because the linear operator in Eq. (23) is not Hermitian, its eigenvalues are complex in general.

For our purposes here we specifically want to find those solutions, if any, that display exponential growth in the $z$ direction (sometimes accompanied with sinusoidal oscillations) and that are therefore unstable. For this task we followed the method described in Ref. 24 by using a Crank-Nicholson scheme. Namely, we solved Eq. (23) taking an arbitrary initial condition, which is assumed to contain any perturbation eigenmode. However, for large propagation distances, the perturbation eigenmode with the largest growth rate will dominate since the growth is exponential. In this way we are able to find the most unstable eigenfunction and the real part of its corresponding eigenvalue, which we call the perturbation growth rate. The grid size was chosen to have as many as 8000 points in the $t$-axis (usually 2000), and the step size in the $z$ direction was typically $\Delta z=0.1$, but was chosen much smaller when the resulting growth rate was high. Numerical accuracy was checked by repetition of the simulations for different grid and step sizes. A typical example of the calculated perturbation eigenmode for a given set of parameters is shown in Fig. 3. Specifically the chosen parameters were $\beta=0.5, \nu=-0.5, \epsilon=0.6, \delta=-0.1$, and $d=d_{-}$. The figure shows the real and the imaginary parts of the eigenfunction; the dashed curve shows the modulus of the stationary but unstable pulse itself. In this case the eigenmode with the highest growth rate is an even function of $t$.

To corroborate the findings of the linear stability analysis, we also solved the nonlinear propagation Eq. (1) by

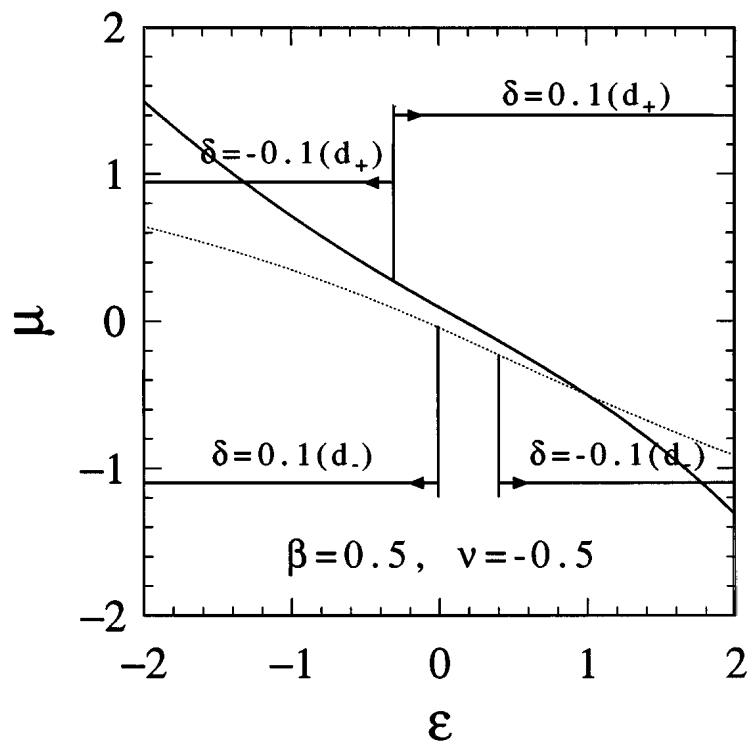

Fig. 2. Dependence of $\mu$ on $\epsilon$ [Eq. (9a)] that must be satisfied as a necessary condition for the general solution to exist when $\beta=0.5$ and $\nu=-0.5$. The solid curve is for $d=d_{+}$in Eq. (10), and the dotted curve is for $d=d_{-}$. The horizontal lines mark the intervals at which the solution given by Eqs. (2)-(17) exists for a given value of $\delta$, which is written on the lines.

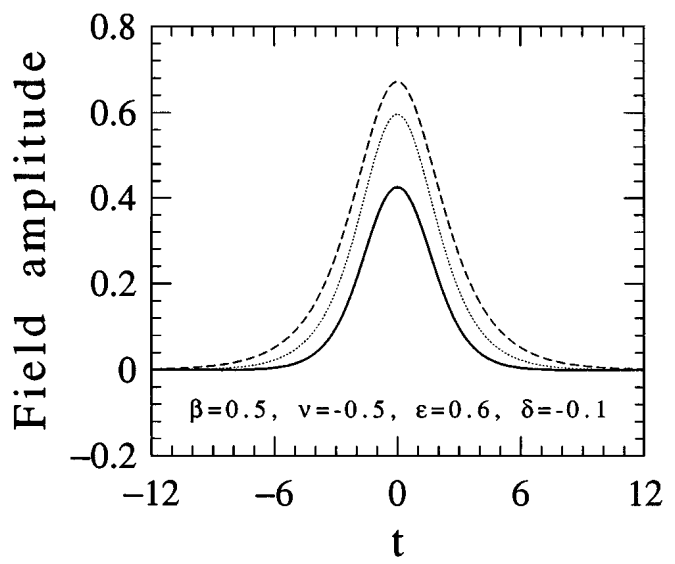

Fig. 3. Field amplitude of the exact solution (dashed curve) for $\beta=0.5, \nu=-0.5, \epsilon=0.6$, and $\delta=-0.1$; the real (solid curve) and the imaginary (dotted curve) parts of the perturbation eigenmode associated with this solution. 
using a split-step Fourier method for several initial conditions. The agreement between the two approaches was total in all the cases we compared.

As an example, Fig. 4 shows the nonlinear evolution of the unstable pulse represented in Fig. 3. In these numerical simulations, instead of the exact eigenmode of perturbation, we added a random perturbation to the stationary solution and took this perturbed solution as the initial condition. Clearly, the random perturbation contains the eigenmode that is shown in Fig. 3, but with unknown sign. Depending on this sign, we can observe two types of evolution. They are shown in Figs. 4(a) and 4(b), respectively. Only these two types of evolution were observed, whatever the perturbation was. When, instead of a random perturbation, we either added to or subtracted from the stationary solution its corresponding eigenmode of perturbation multiplied by a small factor, we obtained exactly the same two types of evolution that Fig. 4 shows. After propagating a certain distance unaltered, the pulse either increases its energy and transforms into uniformly translating fronts [Fig. 4(a)] or decreases and finally disappears [Fig. 4(b)]. This is a typical example of the unstable pulse evolution. In principle, this behavior may slightly change depending on the values of the parameters of the quintic CGLE, but there are always two types of evolution of the unstable exact solutions. In this sense we can consider the exact solutions as boundaries between two different types of behavior of arbitrary pulses. This makes them important in a general analysis.

We now present the results of the linear perturbative analysis applied systematically to the analytical solutions. We consider separately the cases with negative $\nu$ from those with positive $\nu$, as we noticed that they exhibited different characteristics.

\section{A. Case i: $\nu<0$}

Figure 5 shows in a logarithmic scale the perturbation growth rate as a function of $\epsilon$ for $(\beta, \nu)=(0.5,-0.5)$, and for $\delta= \pm 0.1$ and $-\delta= \pm 0.001$. Figure 5(a) is for the cases with negative $\delta$, and Fig. $5(\mathrm{~b})$ is for the positive ones. The value of $\delta$ is written near the respective curves. For each value of $\delta$ there are two curves corresponding to the two possible solutions associated with the two values of $d$. For $|\delta|=0.001$ we have the solid $\left(d=d_{-}\right)$and the dotted curves $\left(d=d_{+}\right)$, and for $|\delta|=0.1$ the dotted-dashed and the dashed curves correspond to $d=d_{-}$and $d=d_{+}$, respectively. Clearly, in all cases the solutions obtained for $d=d_{+}$have much higher instability growth rates than those associated with $d_{-}$. Consequently these last solutions are, in principle, of more interest from a practical point of view. Moreover, as given below, those for negative $\delta$ are connected with the stable solutions.

For positive $\delta$ and for the solutions obtained taking $d=d_{-}$, the figure shows that the perturbation growth rate is exactly equal to $\delta$; that is, their instability has its origin solely in the instability of the uniform background $\psi=0$. The result shows that the pulse itself is stable. This is exactly the case of what takes place in optical transmission lines. ${ }^{5}$ The excess gain is usually positive in order to amplify the pulse itself, but it is kept small to avoid an appreciable growth of the background. What Fig. 5(a) indicates then is that we can have stable propagation of these states for long distances as far as the excess linear gain $\delta$ is low enough. Figure 6 proves this last assertion. We took as an initial condition the following:

$$
\psi(t, 0)=A_{0}(t)[1+\Gamma(t)],
$$

where $A_{0}(t)$ is the corresponding stationary solution [Eq. (3)] for the following coefficients: $\beta=0.5, \nu=-0.5$, $\epsilon=0.1, \delta=0.005$, and $d=d_{-}(\rightarrow \mu=-0.089)$, and $\Gamma$ is a uniform random noise obeying

$$
<\Gamma(t)>=0, \quad \sqrt{<|\Gamma(t)|^{2}>}=0.2 .
$$

The noise term is intended to seed any latent instability of the system, as it surely contains all the possible exponentially growing eigenfunctions of the linearized problem. Figure 6 shows that the solution reaches very quickly the profile corresponding to the stationary solution, getting rid of the random fluctuations, and then remains with it during long distances. After propagating a distance of $z=1000$, the profile has not experienced any relevant change. Farther on, the growth of the background starts to be perceptible.

For negative $\delta$ (and $d=d_{-}$) at any given $\epsilon$ the perturbation growth rate decreases as $|\delta|$ decreases. Another interesting feature of the growth rate curves in Fig. 5(a) is that at $d=d_{-}$the solution becomes more stable as we move to its smallest allowed value of $\epsilon$. This happens when $f_{1}$ becomes close to $f_{2}$. Figure 7 shows an example of the stable propagation found from the perturbative analysis for these cases. We took as initial conditions Eqs. (24) and (25), with $A_{0}(t)$ being the stationary solution for the following values of the coefficients: $\beta=0.5, \nu=-0.5, \delta=-0.1, \epsilon=0.4006$, and
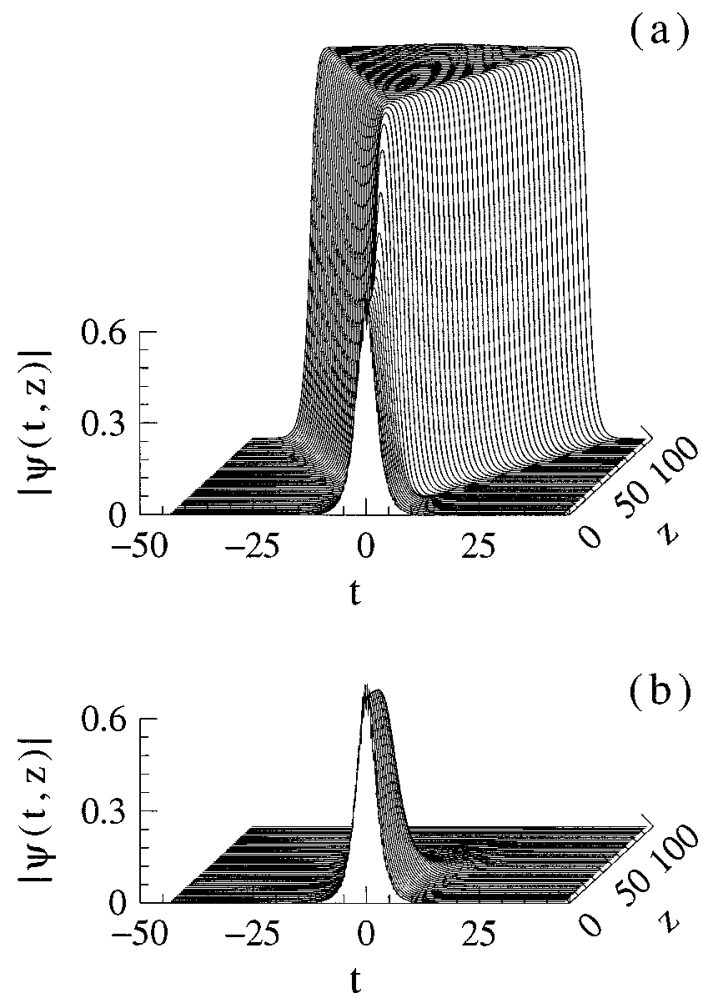

Fig. 4. Two possible scenarios of evolution of the stationary pulse represented in Fig. 3. 

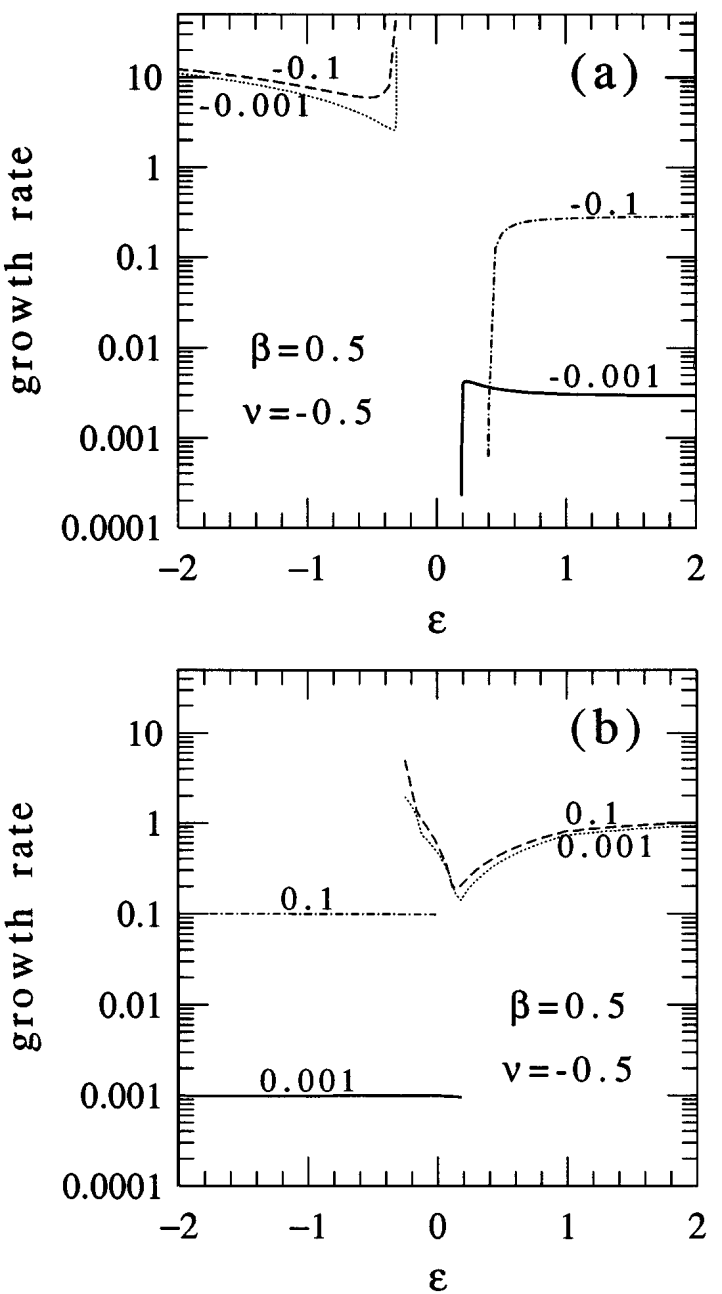

Fig. 5. Growth rate of the predominant perturbation eigenmode associated with the soliton solution as a function of $\epsilon$ for $\beta=0.5$, $\nu=-0.5$, (a) $\delta<0$, and (b) $\delta>0:$ In both cases the solid curve is for $|\delta|=0.001$ and $d=d_{-}$, the dotted-dashed curve is for $|\delta|=0.1$ and $d=d_{-}$, the dotted curve is for $|\delta|=0.001$ and $d=d_{+}$, and the dashed curve is for $|\delta|=0.1$ and $d=d_{+}$. For clarity, the value of $\delta$ is on the corresponding curves.

$d=d_{-}(\rightarrow \mu=-0.227)$. For this specific case, $f_{1}=1.458$ and $f_{2}=1.469$. Initially the solution recovers its unperturbed shape and then propagates during all the distance that we monitored $\left(z_{\max }=10000\right)$ without modifying its profile. We conclude that solutions obtained for negative $\delta$ and $d=d_{-}$are stable when we are in the vicinity of having $f_{1}=f_{2}$ (dotted-dashed and dotted curves in Fig. 1). The transformation of the pulses into a pair of zero-velocity fronts happens on these curves.

We obtained similar results on the stability of these solutions for other values of $\beta$ and $\nu$. Figure 8 shows the perturbation growth rate for different solutions with $\nu=$ -0.1 and $\delta= \pm 0.01$ and with $\beta=0.1$ and $\beta=0.5$. The corresponding value of $\beta$ is written on the curves. The curves for the solution obtained taking $d=d_{+}$are plotted with dotted and dashed curves, and those for $d=d_{-}$are represented in continuous and in dotted-dashed curves. The curves exhibit the same qualitative features as those of Fig. 5.

The general behavior of the perturbation growth rate for other values of $\beta$ and $\nu(<0)$ is similar to that shown in Figs. 5 and 8.
B. Case ii: $\boldsymbol{\nu}>\mathbf{0}$

We also considered the solutions for positive $\nu$. Figure 9 (a) shows the dependence of $\mu$ with respect to $\epsilon$ for $\nu=0.5$ and $\beta=0.1$ (lower curve) and $\beta=0.5$ (upper curve). For $\delta=-0.01$ and $d=d_{-}$the solution exists only for positive values of $\epsilon$, which in addition enforces positive values for $\mu$. That is, the nonlinear gain compensates the linear losses. The regions in which the solution exists is marked in Fig. 9(a) with a solid curve for $\beta=0.5$ and with a dashed curve for $\beta=0.1$. Figure $9(\mathrm{~b})$ shows the perturbation growth rates corresponding to these cases. Two additional curves in Fig. 9(b) are for $\nu=0.1$.

For positive $\nu$, solutions also exist when we take $d=d_{+}$ in Eq. (10), but for very high values of $|\epsilon|$, which also produce high values for $\mu$. For instance, for $\beta=0.5$, $\nu=0.5$, and $\delta=-0.01$, the solution obtained taking $d=$

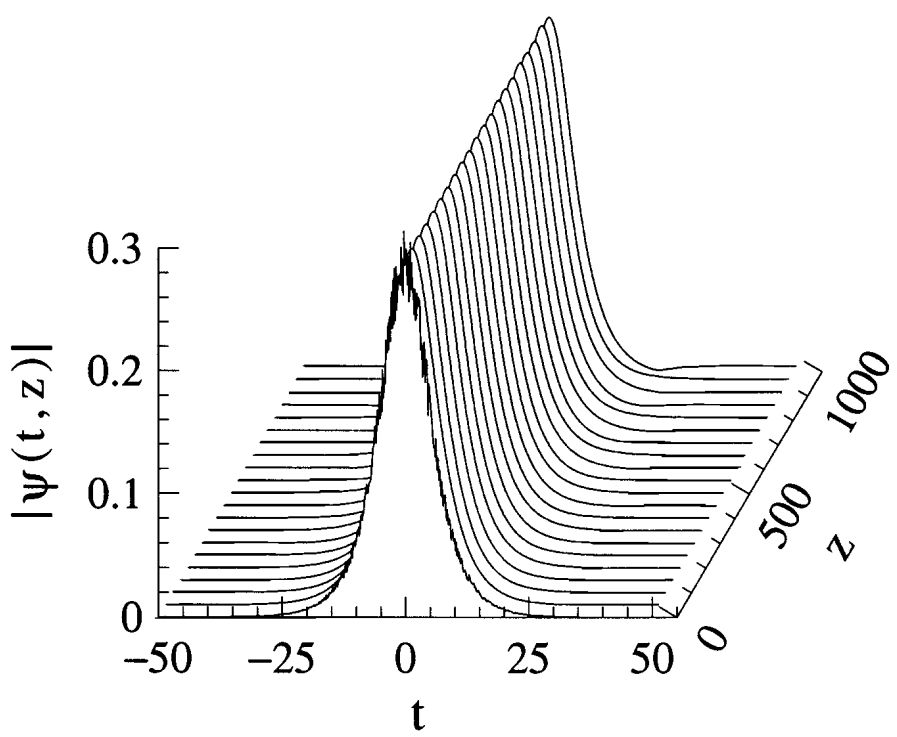

Fig. 6. Evolution of the solution for $\beta=0.5, \epsilon=0.1, \nu=-0.5$, $\delta=+0.005$, and $\mu=-0.09$. This stationary solution is initially perturbed as indicated by Eqs. (24) and (25).

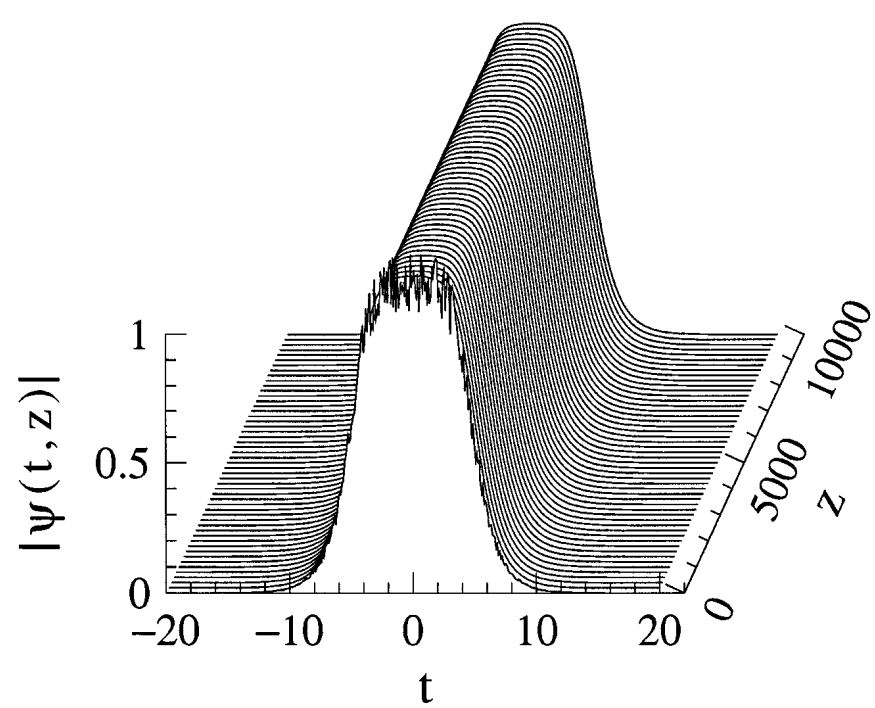

Fig. 7. Evolution of the solution for $\beta=0.5, \epsilon=0.4006$, $\nu=-0.5, \delta=-0.1$, and $\mu=-0.227$. This stationary solution is initially perturbed as indicated by Eqs. (24) and (25). 

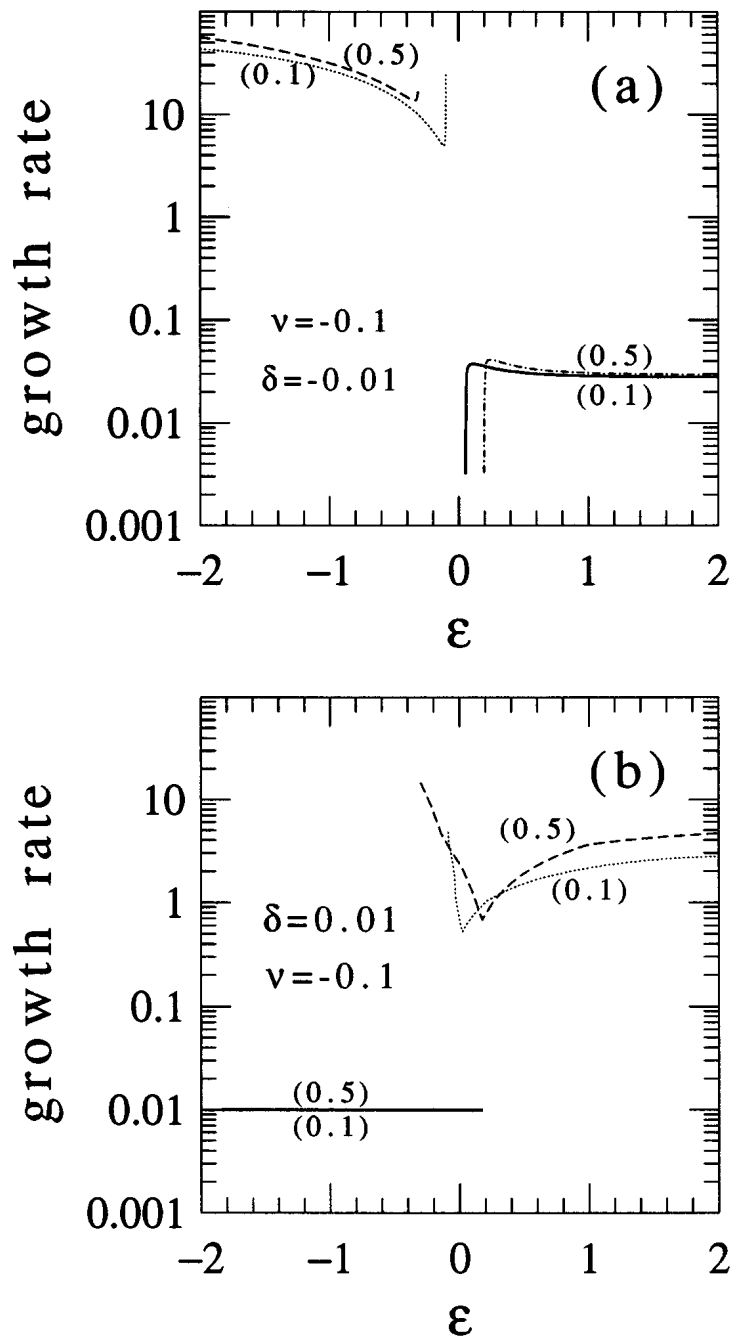

Fig. 8. Growth rate of the predominant perturbation eigenmode associated with the soliton solution as a function of $\epsilon$ for $\nu=-0.1, \beta=0.5, \beta=0.1$, (a) $\delta=-0.01$, and (b) $\delta=-0.05$. The different curves correspond to (i) $\beta=0.5$ and $d=d_{-}$ (solid curves), (ii) $\beta=0.1$ and $d=d_{-}$(dotted-dashed curves), (iii) $\beta=0.5$ and $d=d_{+}$(dashed curves), (iv) $\beta=0.1$ and $d=d_{+}$(dotted curves). For clarity we wrote the values of $\beta$ in parentheses near the corresponding curves.

$d_{+}$exists in the interval $(-\infty,-9.93)$, and, for example, for $\epsilon=-12, \mu$ becomes 32.97. These values are quite unrealistic. Moreover, we found that the corresponding solutions are highly unstable.

The general conclusion from the above stability analysis is that, although an exact solution (17) to the quintic CGLE can be found when a specific relation between the parameters in Eq. (9a) is satisfied, all of them are unstable. An exception appears in the vicinity of the boundary that separates pulses from pairs of fronts. The perturbation growth rate of these soliton solutions falls to zero when we tend to this limit. These stable solutions have a flat top, announcing the transition from pulses to fronts.

\section{REGIONS IN THE PARAMETER SPACE IN WHICH STABLE PULSES EXIST}

In this section we obtain numerically the values of the coefficients $(\delta, \beta, \epsilon, \mu, \nu)$ of the quintic CGLE (subspace of the parameter space) for which stable pulses exist. We find stable pulses in a certain region and compare it with another (of a lower dimensionality) in which the analytical solutions given by Eq. (17) exist.

Let us first fix some limits in the parameter space in which to look for stable pulses. The parameter $\beta$ clearly must be nonnegative in order to stabilize the soliton in the frequency domain. The linear gain coefficient $\delta$ must be zero or negative to provide the stability of the background. In this case, for $\nu=0$, stable pulses can exist only for $\epsilon$ above the line $S$. We choose $\mu<0$ to stabilize the pulse against the collapse. The parameter $\nu$ can have either sign.

With these restrictions in mind we chose a given value for the coefficients and solved numerically the propagation Eq. (1) taking as the initial condition a Gaussian pulse of arbitrary amplitude and width. The shape of the initial pulse appeared of minor importance. Then we
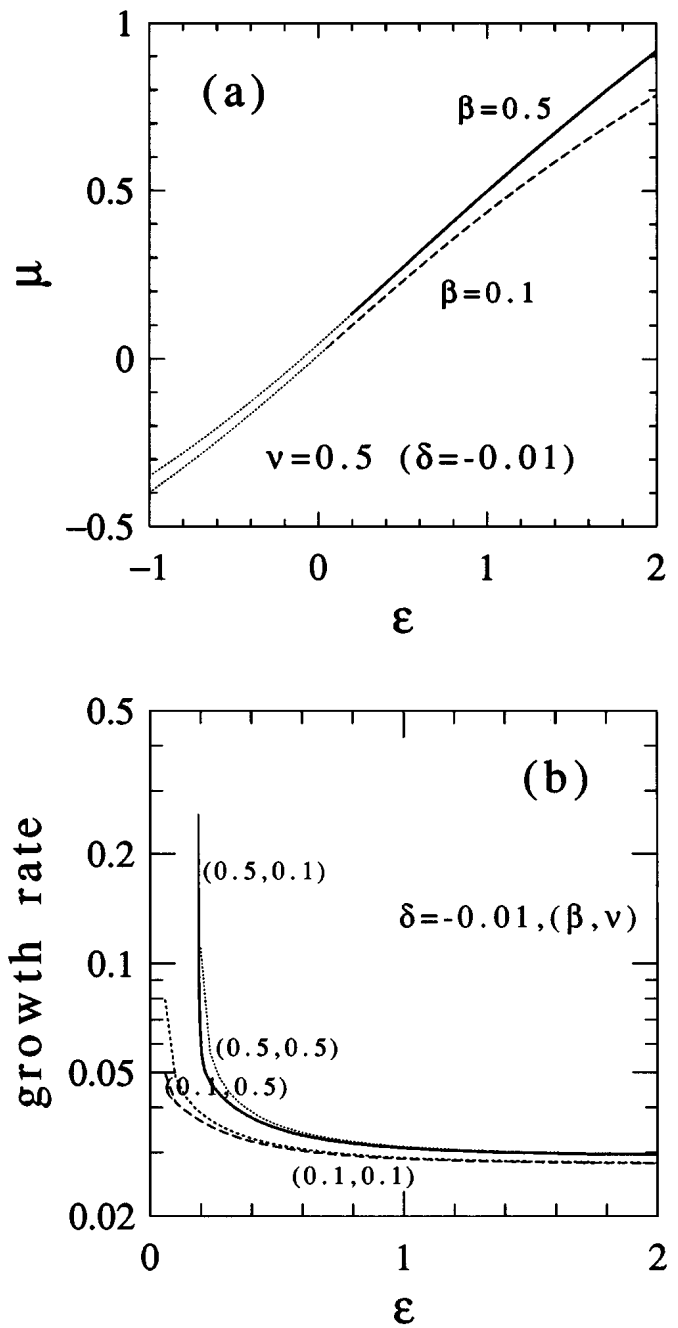

Fig. 9. (a) Dependence of $\mu$ on $\epsilon$ [Eq. (9a)] that must be satisfied as a necessary condition for the general solution Eq. (21) to exist when $\nu=-0.5$ and $\beta=0.5$ (upper curve) and $\beta=0.1$ (lower curve). The solid and the dashed parts of these curves show the regions in which the solutions actually exists for $\delta=-0.01$. (b) The growth rate of the predominant perturbation eigenmode associated with the soliton solution as a function of $\epsilon$ for $\delta=-0.01$ and $(\beta, \nu)$ equal to (i) $(0.5,0.1)$ (solid curve), (ii) $(0.5,0.5)$ (dotted curve), (iii) $(0.1,0.1)$ (long-dashed curve), and (iv) $(0.1,0.5)$ (short-dashed curve). 
observed its evolution upon propagation. If the solution converged to a stationary one, we considered that we had obtained a stable solution and that the chosen values of the parameters belong to the class of those that permit the existence of solitons. Figure 10 shows three examples of the soliton solutions found with the above method. The corresponding values of the coefficients are $\beta=0.5$, $\delta=\nu=\mu=-0.1, \epsilon=0.38$ (solid curve), $\epsilon=0.52$ (dotted curve), and $\epsilon=0.66$ (dashed curve).

Repeating these calculations systematically for other sets of parameters, we were able to construct the areas in the parameter space in which stable propagation of bounded solutions is possible. The process of convergence to a stable solution was usually quite slow, but we found that it could be accelerated in the following manner. After having found a stable solution, we took it as the initial condition for the next case, in which we kept constant all the parameters except one (usually $\epsilon$ ), which was slightly changed. The point is that, as expected, for a similar set of parameters we obtained similar solutions, and therefore we had a rapid convergence.

Figure 11 shows the areas in the $(\beta, \epsilon)$ plane where soliton solutions were found numerically. The differently hatched areas correspond to different values of the parameter $\mu$. The lower curve (dashed) represents the curve $S$ [Eq. (16)], and it is plotted to allow us to make some comparisons with the conclusions we obtained concerning the analytic solutions. First, the region of stable pulses is always above the curve $S$, and the lower boundary of the stability region (solid curve) is approximately parallel to the curve $S$. The distance between this lower boundary and the curve $S$ depends on $\delta, \mu$, and $\nu$. For small $\mu, \nu$, and $\delta$ this distance is small. This result cannot be obtained by application of perturbative theory to the soliton solutions of the nonlinear Schrödinger equations, because $\beta$ and $\epsilon$ are not small. For given values of $\nu$ and $\delta$ the hatched regions become wider as $|\mu|$ increases, and its lower boundary becomes higher. For given values of $\nu$ and $\mu$ the lower boundary approaches the curve $S$ as $\delta$ goes to zero. We would expect that at zero $\delta$ the curve $S$ would be the onset of instability. Figures 11(a) and 11(b) give a rough idea of how the regions of existence of stable pulses in the plane $(\beta, \epsilon)$ change when $\mu$ and $\delta$ are changed. We now consider other planes in the parameter space in which we found stable pulselike solutions.

Figure 12 shows the region of stable pulses in the plane $(\nu, \epsilon)$ for fixed values of $\mu, \delta$, and $\beta$ as written on the figure. The plot shows that the width of the stripe in Fig. 10 increases largely as $\nu$ increases. The dashed curve in Fig. 12 shows where the exact analytical solutions are located for the same set of parameters. Interestingly enough, this curve is also almost parallel to the upper border of the area of stable pulses but is located some distance from it. This shows that the analytical solutions are beyond that region and are therefore in total agreement with the stability analysis, unstable.

It is also interesting to study how the region in which stable pulses exist depends on $\delta$. Figure 13 shows this dependence for fixed values of $\mu, \nu$, and $\beta$. Specifically, $\nu=\mu=-0.1$ and $\beta=-0.5$. As the linear excess gain decreases, the interval of allowed values of $\epsilon$ increases, and its central value increases as $\delta$ increases, which logically means that larger linear losses must be compensated (if the rest of the parameters are constant) by an increase in the third-order nonlinear gain. For the above values of $\nu, \mu$, and $\beta$, Eqs. (9) give $\epsilon=1$; that is, as expected, above the hatched region.

Finally, Fig. 14 shows the area of stable pulses in the plane $(\mu, \epsilon)$ for fixed values of $\nu, \delta$, and $\beta$. As $|\mu|$ increases, the interval of allowed values of $\epsilon$ becomes wider, and its central value becomes larger. This last observation is also expected, as it indicates that larger fifth-order nonlinear losses must be compensated by an increase in the third-order nonlinear gain. The width of the stripe becomes infinitesimally small at $\mu \approx-0.04$. The dashed curve represents the points at which the exact analytical solutions are located for the chosen values of $(\delta, \nu, \beta)=(-0.1,-0.1,0.5)$. Again, it can be seen that they are out of the area of stable pulses. However, in this case the distance between the region of stable pulses and the exact analytical solutions increases with $\mu$ and goes to zero at $\mu \rightarrow-0.04$. The instability growth rate of the corresponding analytical solution for this value of $\mu$ becomes negligible.
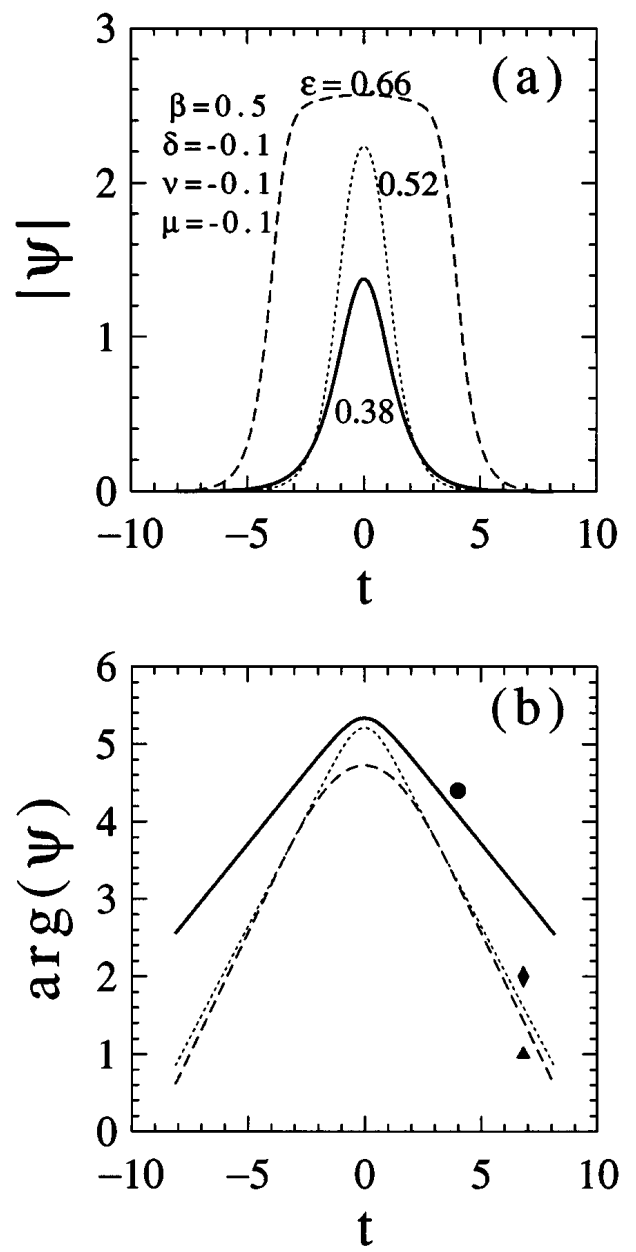

Fig. 10. Numerically found soliton solutions for $\beta=0.5$, $\nu=\mu=\delta=-0.1, \epsilon=0.38$ (solid curves), $\epsilon=0.52$ (dotted curves) and $\epsilon=0.66$ (dashed curves). (a) Amplitude profile $|\psi|$, (b) phase profile $\arg (\psi)$. The circle, the diamond, and the triangle symbols associated with the cases $\epsilon$ equal to $0.38,0.52$, and 0.66 , respectively, are used in the following figures to locate these solutions in the parameter space. 

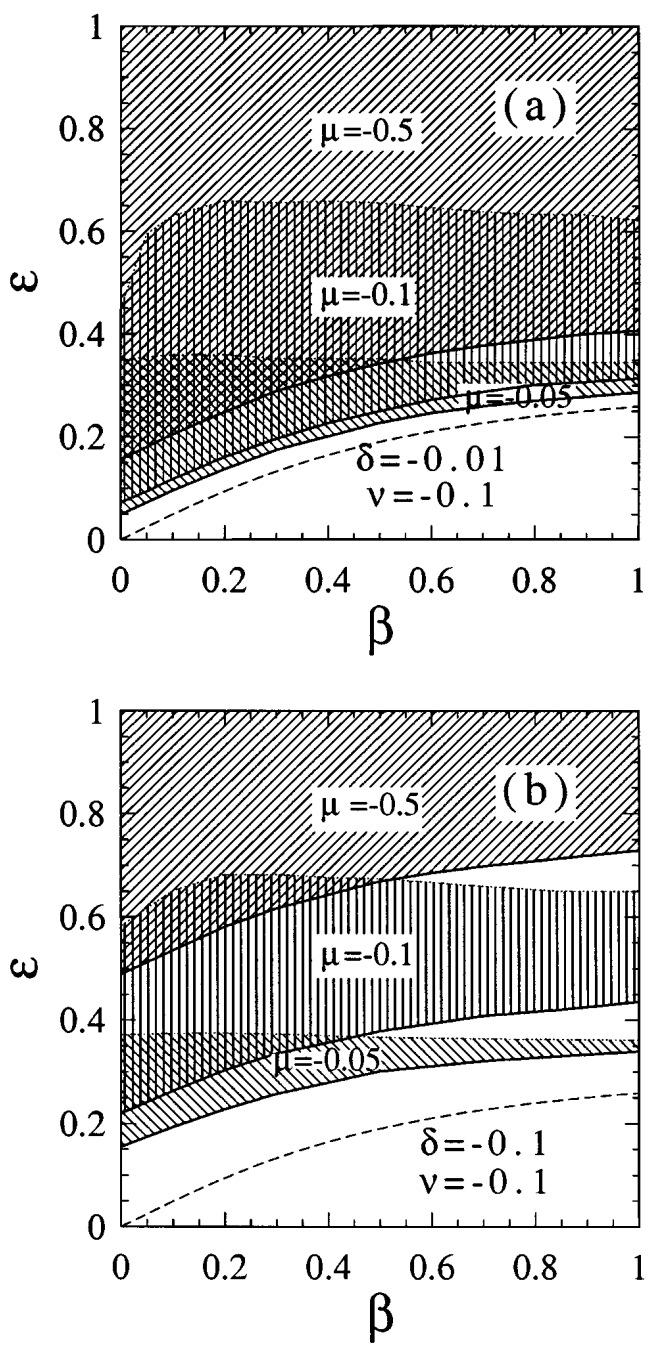

Fig. 11. Region in the $(\beta-\epsilon)$ plane in which stable pulselike solutions are found. Differently hatched areas are for different values of $\mu$, which are written in the corresponding regions. All these areas are located above the curves $S$ (dashed curves). (a) $\delta=-0.01, \nu=-0.1$, and (b) $\delta=\nu=-0.1$.

\section{DISCUSSION}

The propagation dynamics outside the region of existence of stable pulses depends on the parameters of the equation as well as on the initial pulse shape. Its detailed study is beyond the scope of this paper. However, we can extract some general behaviors and explain them in relation to our above results. Clearly, stationary pulses must balance losses and gain. For systems whose parameters are located below the lower boundary of our hatched regions, pulses damp down as they propagate. The energy flux provided to the initial pulse owing to positive $\epsilon$ is less than the energy decrease owing to linear $(\beta>0, \delta<0)$ and nonlinear $(\mu<0)$ losses. The physical processes on the upper boundary are different. Generally the upper boundary in the $(\beta, \epsilon)$ diagram coincides with the line at which fronts have zero velocity. Above this curve, two fronts of the wide pulse diverge from each other, and below the curve two fronts converge to each other, forming a stable pulse at the end of this process. Hence, stable pulses can exist only below this curve.
In all these simulations the curves in the parameter space at which the analytical solutions exist were outside the region in which we numerically found stable pulses. This agrees with our stability analysis of the exact analytical solutions in Section 3 and indicates the need of further analytical developments. Our exact analytical solutions can serve as a basis for this task. Further generalizations can be done exactly with a more general ansatz for the pulse chirp than Eq. (5) or approximately with our solutions as zeroth-order approximation.

The first observation of strictly stable pulselike solutions has been reported by Thual and Fauve. ${ }^{25}$ They found some points in the parameter space at which stable pulses exist. Rough estimates of the location of the boundaries between fronts and pulselike solutions of CGLE have been done by Hakim, Jacobsen, and Pomeau. $^{26}$ However, there is not a sharp boundary between the two classes of solutions. It has been found by

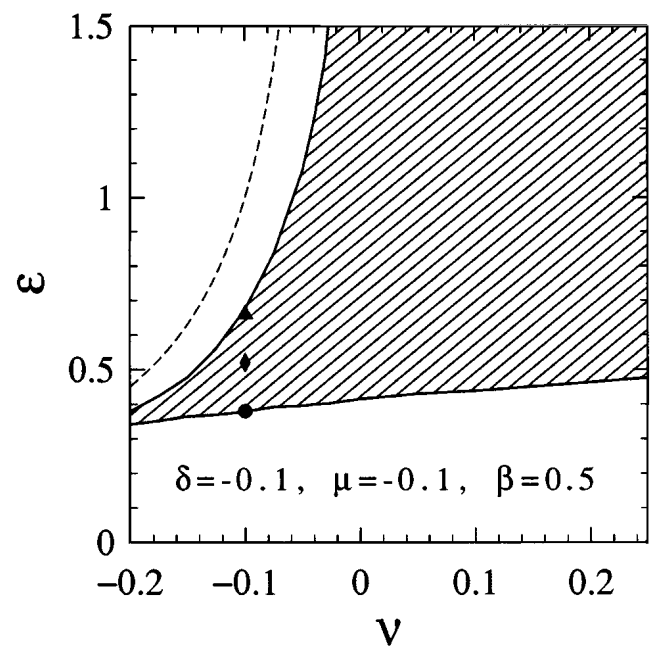

Fig. 12. Region in the plane $(\nu, \epsilon)$ for which stable pulses are possible. $\beta=0.5$ and $\mu=\delta=-0.1$. The dashed curve represents the points at which the analytical solution given by Eq. (17) exists. The three symbols (circle, diamond, and triangle) show the locations of the solutions represented in Fig. 10.

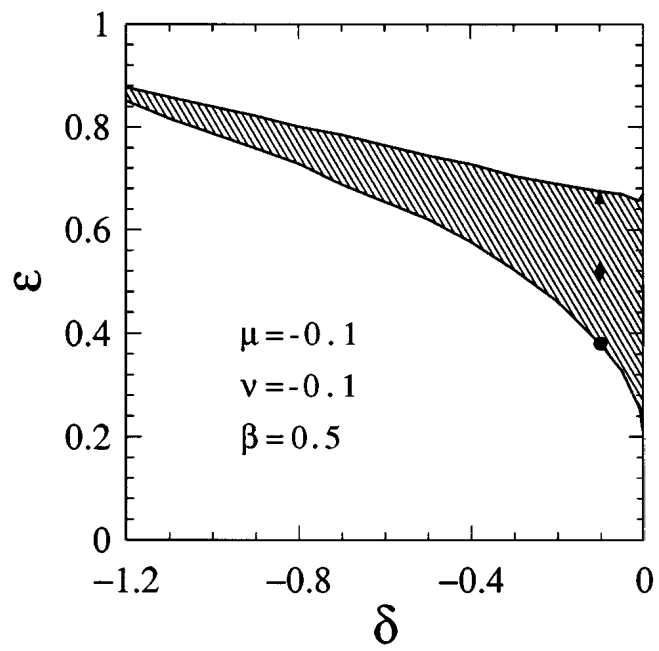

Fig. 13. Region in the plane $(\delta, \epsilon)$ in which stable pulses are possible. $\beta=0.5$ and $\nu=\mu=-0.1$. For these parameters the analytical solution exists at $\epsilon=1$. The three symbols (circle, diamond, and triangle) show the locations of the solutions represented in Fig. 10. 


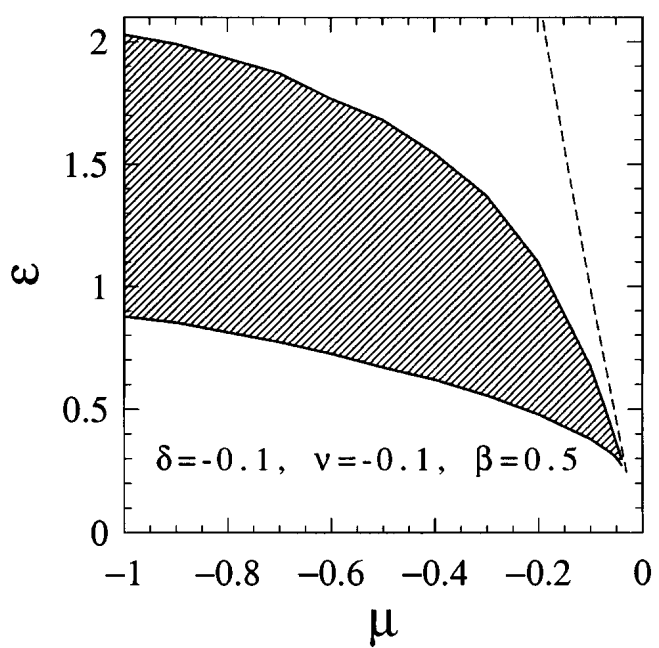

Fig. 14. Region in the plane $(\mu, \epsilon)$ in which stable pulses are found. $\beta=0.5$ and $\nu=\delta=-0.1$. The dashed line represents the points at which the analytical solution exists.

van Saarlos and Hohenberg ${ }^{20}$ that, for given values of the parameters, a variety of fronts and pulselike solutions exist. This agrees with our observations, in which we found that pulses and positive velocity fronts coexist in some tiny region near the upper boundary.

Clearly, stable pulses are the most interesting objects in optics. They are produced by laser systems and they constitute bits of information, transmitted in optical fiber systems. Hence it is important to know where in the parameter space we can expect stable pulses. In this study we find those regions and, additionally, show that exact analytic solutions known to date are beyond these regions. These results can be used for the analysis of a fiber laser with pulse mode locking, ${ }^{14}$ for additive pulse limiting, ${ }^{27}$ and for the analysis of optical transmission lines with spectral filtering. ${ }^{28}$

\section{CONCLUSIONS}

We have studied the stability of analytic solutions of the quintic complex Ginzburg-Landau equation. These solutions exist when the values of the coefficients of the CGLE are related by a certain equation. For most of the values of the coefficients we have found that these solutions were unstable, and we have calculated their corresponding perturbation growth rates. We have also numerically found the regions in the parameter space in which stable pulse propagation takes place and have compared them with the areas of existence of exact analytical solutions.

\section{ACKNOWLEDGMENTS}

The work of J. M. Soto-Crespo was supported by the Comisión Interministerial de Ciencia y Tecnología under contract TIC95-0563-C05-03. The work of N. N. Akhmediev and V. V. Afanasjev is supported by the Australian Photonics Cooperative Research Centre.

\section{REFERENCES}

1. J. D. Moores, "On the Ginzburg-Landau laser mode-locking model with fifth-order saturable absorber term," Opt. Commun. 96, 65 (1993).

2. E. P. Ippen, H. A. Haus, and L. Y. Liu, "Additive pulse mode locking," J. Opt. Soc. Am. B 6, 1736 (1989).

3. C.-J. Chen, P. K. A. Wai, and C. R. Menyuk, "Stability of passively mode-locked fiber lasers with fast saturable absorption," Opt. Lett. 19, 198 (1994); "Self-starting of passively mode-locked lasers with fast saturable absorbers," Opt. Lett. 20, 350 (1995).

4. A. Mekozzi, J. D. Moores, H. A. Haus, and Y. Lai, "Soliton transmission control," Opt. Lett. 16, 1841 (1991); "Modulation and filtering control of soliton transmission," J. Opt. Soc. Am. B 9, 1350 (1992).

5. Y. Kodama and A. Hasegawa, "Generation of asymptotically stable optical solitons and suppression of the Gordon-Haus effect," Opt. Lett. 17, 31 (1992).

6. M. Romagnoli, S. Wabnitz, and M. Midrio, "Bandwidth limits of soliton transmission with sliding filters," Opt. Commun 104, 293 (1994).

7. V. V. Afanasjev, "Interpretation of the effect of reduction of soliton interaction by bandwidth-limited amplification," Opt. Lett. 18, 790 (1993); "Soliton singularity in the system with nonlinear gain," Opt. Lett. 20, 704 (1995).

8. D. Atkinson, W. Loh, V. V. Afanasjev, A. B. Grudinin, A. J. Seeds, and D. N. Payne, "Increased amplifier spacing in a soliton system with quantum-well saturable absorbers and spectral filtering," Opt. Lett. 19, 1514 (1994).

9. M. Matusmoto, H. Ikeda, T. Uda, and A. Hasegawa, "Stable soliton transmission in the system with nonlinear gain," J. Lightwave Technol. 13, 658 (1995).

10. G. P. Agrawal, "Optical pulse propagation in doped fiber amplifier," Phys. Rev. A 44, 7495 (1991); "Effect of twophoton absorption on the amplification of ultrashort optical pulses," Phys. Rev. E 48, 2316 (1993).

11. R. Graham, in Fluctuations, Instabilities, and Phase Transitions, T. Riste, ed. (Springer, New York, 1975).

12. J. A. Powell, A. C. Newell, and C. K. R. T. Jones, "Competition between generic and nongeneric fronts in envelope equations," Phys. Rev. A 44, 3636 (1991).

13. M. J. Landman, "Solutions of the Ginzburg-Landau equation of interest in shear flow transition," Stud. Appl. Math 76, 187 (1987).

14. H. A. Haus, J. G. Fujimoto, and E. P. Ippen, "Structures for additive pulse mode locking," J. Opt. Soc. Am. B 8, 2068 (1991).

15. L. Gagnon and P. A. Bélanger, "Adiabatic amplification of optical solitons," Phys. Rev. A 41, 6187 (1991).

16. J. D. Moores, W. S. Wong, and H. A. Haus, "Stability and timing maintenance in soliton transmission and storage rings," Opt. Commun. 113, 153 (1994).

17. F. I. Khatri, J. D. Moores, G. Lenz, and H. A. Haus, "Models for self-limited additive pulse mode-locking," Opt. Commun. 114, 447 (1995)

18. C. Paré, L. Gagnon, and P. A. Bélanger, "Spatial solitary wave in a weakly saturated amplifying/absorbing medium," Opt. Commun. 74, 228 (1989).

19. W. van Saarloos and P. C. Hohenberg, "Pulses and fronts in the complex Ginzburg-Landau equation near a subcritical bifurcation," Phys. Rev. Lett. 64, 749 (1990).

20. W. van Saarloos and P. C. Hohenberg, "Fronts, pulses, sources and sinks in generalized complex Ginzburg-Landau equations," Physica D 56, 303 (1992).

21. P. Marcq, H. Chaté, and R. Conte, "Exact solutions of the one-dimensional quintic complex Ginzburg-Landau equation," Physica D 73, 305 (1994).

22. R. Conte and M. Musette, "Linearity inside nonlinearity: exact solutions to the complex Ginzburg-Landau equation," Physica D 69, 1 (1993).

23. N. N. Akhmediev, V. M. Eleonskii, and N. E. Kulagin, "Exact first order solutions of the nonlinear Schrödinger equation," Theor. Math. Phys. (USSR) 72, 809 (1987).

24. N. N. Akhmediev, V. I. Korneev, and Yu. V. Kuz'menko, "Excitation of nonlinear surface waves by Gaussian light 
beams," Zh. Eksp. Teor. Fiz 88, 107 (1985) [Sov. Phys. JETP 61, 62 (1985)].

25. S. Fauve and O. Thual, "Solitary waves generated by subcritical instabilities in dissipative systems," Phys. Rev. Lett. 64, 282 (1990); O. Thual and S. Fauve, "Localized structures generated by subcritical instabilities," J. Phys. (Paris) 49, 1829 (1988)

26. V. Hakim, P. Jakobsen, and Y. Pomeau, "Front versus soli- tary waves in nonequilibrium systems," Europhys. Lett. 11, 19 (1990).

27. C. R. Doerr, H. A. Haus, and E. P. Ippen, "Additive-pulse limiting," Opt. Lett. 19, 31 (1994).

28. L. F. Mollenauer, J. P. Gordon, and S. G. Evangelides, "Sliding-frequency guiding filter: an improved form of soliton jitter control," Opt. Lett. 17, 1575 (1992). 